prospects for success. When meteorism develops and bowel movement is not produced by the usual enema and other remedies, every hour lost with the use of turpentine, alum, asafetida and other enemas merely weakens the reactive power of the bowel and renders regeneration by means of the electric current more ditlicult.

The battery used in these cases consists of a twentyfour dry-cell apparatus with galvanic and faradic (high tension) attachments, rheostat and milammeter, and is readily portable to the bedside.

1748 Broadway.

\section{BACILLUS AEROGENES CAPSULATUS IXFEC- TIONS, WITH REPORT OF TEN CASES}

\section{WALTER R. HEWITT, M.D.}

Resident Physician. St. Louis (ity Hospital

$$
\text { st. LOUIS }
$$

This infection is interesting because of the high death-rate and the frequency with which the condition is overlooked until too late for surgical interference. It is looked on as a rare condition, though it is more frequent than one would suppose. There have been three cases at the City Hospital since June 1, 1910, with three recoveries.

To Maissonneuve and Pirogoff is due the credit for the first clinical descriptions of the disease. Maissonneuve, writing in 1853 , called the disease gangrène foudroyante, but it was not until 1891 that Dr. Welch discovered and described the organism. There has been much confusion as regards this organism and its pathologic processes, even the latest text-books giving little space to a clear description of this definite infection and its danger to life.

The most frequent sources from which the organisms may be obtained are the intestinal tracts of mammals and the soil, although Welch (1896) recovered it from a bullet in the head of the tibia.

The organism (Bacillus aerogenes capsulatus) normally grows in the absence of oxygen, although Lahey ${ }^{-1}$ demonstrated that the $B$. a. c. may grow on culture media in the presence of oxygen.

\section{SYMPTOMS}

There seem to be three principal types of the infection; namely, simple, fulminating, and mixed.

The incubation period, as nearly as could be ascertained in ten cases, was forty-eight hours. In one case it was twenty-one hours (Case 10). In the simple cases, there is slight rise in temperature and pulse, the patient appears seriously ill, and there is a dark, almost black, bloody discharge, which resembles a thin syrup. This discharge usually brings out a few small bubbles, and leaves us wondering what infection is present. Pus is not present.

The fulminating or malignant type depends on the severity, virulence and site of the infection, and not only affords the best opportunities for study of patholngic processes, but soon convinces one that something rarlical must be done and done quickly. In this type the patient is usually found with high temperature (103-10+ F.) and rapid pulse (130-150), with rapid respiration (3040 per minute), and appears critically ill. B.a.c. infec-

1. Blake. J. B., and Iahey, F. II. : Infections Due to the Bacillus Aerogenes Capsulatus, The Journal A. M. A., May 21, 1911, p. if: 1 . tion occurs most ofen after injurjes in which tissues are pulpified and very dirty. About forty-eight hours afterward, when the wound is dressed, a dark, thin, syrupy, foul-smelling discharge escapes with gas bubbles.

The fulminating type on the leg may take on the appearance of an erysipelatous condition, though without raised borders, there being a dark or brownish discoloration. This was the condition in Case $\%$, plus a lymphangitis, which was at first deceptive. Blebs of variable sizes are frequently present and soon take on a blue or greenish appearance. When opened, the characteristic, foul-smelling fuid with its sweetish odor, becomes apparent. If an extremity is involved, it rapidly becomes gangrenous and distended with gas of a composition of hrdrogen 64.3, carbon dioxid $2 \% .6$ and nitrogen 8.1 (burning with a pale blue flame). This was the clue to the diagnosis in Case 9, althongh the diagnosis was suspected from the early rise in temperature (first twenty-one hours) and the history of injurv. Cultures will show the presence of organism, but with a clear rinical picture operation should be undertaken at once.

Pain was absent in all except Case 10. Here the patient's pain became very great, and as he was not furnished relief by morphin. after three attempts to get consent to operation had failed, he finally consented to anputation, which was done, and he recovered. (Blake and Lahey explain the absence of pain on the ground of the necrosing action of $B . a, c$. interfering with nerve conductivity, although the presence of gas and distention, as I believe, with its mechanical effect. must not be forgotten. A sort of anesthesia is produced.)

Delirium occurs late, after the general infection, and persists till death. In Case 4 , delirium was so great that diagnosis of delirium tremens'and infection was made.

Within seventy-two hours. toxemia is pronounced, and I agree with Blake and Lahey that recovery is not the rule after the third dav.

Nausea and vomiting occur late, and, like delirium, are indicative of generalized infection.

The process extends chiefly by direct extension, as the gas separates and dissects between muscle and fascial planes and follows the lines of least resistance.

The lymph-glands are seldom enlarged and tender, and only when other organisms are present, as the streptococcus (Case 10). The rapidity with which the process ascends a limb probably accounts also in pairt for the frequency of non-involvement of lymph-glands.

Emphrsema or crepitus was elicited in Cases 2, 3, 4, 5, 7, 8 and 9. and in one rase gas escaped with a noise. In Case 1, a tympanitic note was elicited on percussion.

Fascia, tendons and ligaments resist infection strongly. Muscle, especially pulpified muscle, affords a flourishing site for the infection. At the wound site, the tissues are soft, friable and pulplike; while beyond, the tissues are pale and edematous, contain small amounts of gas, and are rapidly being converted into a pulpified state.

Mixed infections, whether streptococcus or staphylococus, in which infection remains at the site of inoculation, are usually mild.

\section{TREATMENT}

Treatment is always uperative unless there is a generalized infection.

In the presence of general infection, the free opening of the wound, with hydrogen peroxid as a dressing should be tried. If a leg or thigh is involved, spina] anesthesia may be used.

There are, however, two recognized methods of treatment-amputation and multiple incisions. Amputation 
is the operation of choice in all malignant cases, as it offers good chance for recovery. What one of us would care to treat such cases with multiple incisions and hydrogen peroxid in view of the fact that oxygen does not always kill the organisms (Lahey) and in these cases the organisms are far from the original site of the wound and hydrogen peroxid cannot reach them. Of course, in a simple case, multiple incision and hydrogen peroxid might be tried, and even oxygen carried into the wound with catheter. Under careful observation if amputation is performed when advance takes place, recovery may be expected.

Of course, an occasional limb may be sacrificed with amputation, but what when we try multiple incisions and find later a dead patient?

In my series of cases, three patients recovered (all in cases of malignant type and developing within fortyeight hours) after amputation, one leg and two arms being sacrificed. In cases recovering under multiple incision and hydrogen peroxid treatment, mighit not there be some other gas-producing organism?

In this series of cases, Patient 3 was of necessity treated with multiple incisions, as the infection involved the trunk. The patient promptly died, as infection was general.

\section{PROPHYLAXIS}

Ordinary antisepties, tincture of iodin, bichlorid, etc., have little or no effect on the organism, as I have seen cases develop after vigorous treatment with these antiseptics.

\section{CASE REPORTS}

CASE 1.-H. H., a carpenter, aged 56 (No. 5776), was admitted Nov. 16, 1906. This patient was accidentally shot November 18 in a tent while on a hunting trip. He was treated by Drs. Taylor and Pickel of Festus, Mo., who forwarded him to St. Louis for treatment. The patient's condition when seen by them was as follows:

"The soft tissues were nearly all shot away just above the ankle. Both bones were badly shattered. The foot was connected with the leg only by a narrow strip of skin and some fragments of muscle. It was not dressed in any way except in the blanket that the patient was lying on. There was no dirt in the wound, more than would naturally get into it from the bedding, which was not very clean. There had been no attempt made to control hemorrhage; consequently he had lost about all that he was able to stand. He was in a state of considerable shock, so that only a trimming of the remaining shreds of tissue was performed, and bleeding points ligated. No attempt made to disinfect the wound, other than to apply a 2.5 per cent. solution of phenol (carbolic acid) and get him to the hospital as soon as possible.

The doctors at Festus were of the opinion that the infection rame from the blanket in which his leg was wrapped.

When he arrived at the St. Louis City Hospital, it was found that he had a ragged amputation of the left leg four inches nbove ankle-joint, with much laceration of soft tissue and comminution of bones. The patient arrived at the hospital at $s: 30$ in the evening, having been injured about eleven in the morning of the same day. Hemorrhage had ceased when he arrived at the hospital. The leg was shaved and cleansed, put in a large saline pack without much effort to trim away the ragged tissues. Patient complained of weakness and thirst; pulse was almost imperceptible: temperature subnormal. Salt solution, by hypodermoclysis was given; external heat; patient placed in bed; antitetanic serum given; condition bad; pulse 120, respirations 24 , temperature $97.2 \mathrm{~F}$. Crinalysis revealed the presence of albumin and a great many hyaline and finely aud coarsely granular casts.

Within twenty-four hours after the accident, amputation was performed in the middle third of the leg, the circular flap method being used. After heing in the ward sixteen hours, a peculiar, stinking, sweetislı odor was noticed about the patient, and on dressing the wound it was found to be discolored. Blood-culture was positive.

On the night of November 18 the patient was beginning to show symptoms of general infection, pulse 150 , respiration 50 , very shallow and mostly abdominal. The patient complained of pain in the abdomen and over the epigastric region. The abdomen was distended and soft, and over the left lower quadrant was a soft, fluctuating crepitus. The same condition was present over the left thigh, but in a more marked degree. The surgical wound with its dressing was not disturbed.

The patient at 3 o'clock in the afternoon complained of great difficulty in breathing. At $3: 25 \mathrm{p}$. m. the patient was sinking rapidly, the heart failing to respond, although he was delirions and trying to get out of bed. The patient lost consciousness about 4 p. m., showing evidences of air-hunger, gasping for air. The heart-beat was not palpable; small, thready pulse. Patient died at 4:22 p. m., November 19, three days aftr. admission.

This was a very virulent infection, traveling up the leg very rapidly. In a few hours, it had extended up as far as the ne.k. death following. Post-mortem findings were as follows:

Gangrenous emphysematous stump, universal emphysema of the subcutaneous tissues, emphysema of the heart, lungs, liver, kidneys and spleen, acute enlargement of the spleen, congestion and edema of the lungs, and a foany condition of organs.

This case is interesting as showing another source of infection: that of a gunshot wound; it also shows that great loss of blood and pulpification of tissue influence an early appearance of the infection on account of the patient having practically no resistance. The case was an early, malignant type with general infection.

CASE 2.-M. N., a laborer, aged 41 (No. 6642), entered Nov. 30,1907 , with a history of having been run over by a locomotive. As a result, both legs from a few inches below the knees to above the shoe-tops were pulpified. The wounds were dirty. An emergency trimming operation was performed. Pulse at this time was 106 , respirations 24 , temperature $99 \mathrm{l}$. Antiseptics used in first-aid treatment were bichlorid, tincture of iodin, and turpentine.

On the morning of December 3, patient developed a high temperature, rapid pulse and signs of severe shoek. Bichlorid pack was applied at this time. On the morning of December 4 , an offensive, characteristic sweetish odor was present. There was some emphysema of the left thigh. The left thigh at 3 p. m. was greatly distended and tympanitic on percussion. At 6 p. m., the left limb was tense with gas, the gas being found subcutaneously between the fascia and muscle and up in the left groin, and it extended in the midaxillary line nearly to the axilla. Numerous incisions were made, and the gas escaped with a noise. At this time, a bichlorid and turpentine dressing was applied, with a diagnosis of emphysematous gangrene. The patient died at $8: 55$ p. m., five days after admission, of a general B. a. c. infection (clinical).

The patient had become very delirious on the evening of December 3 , and delirium persisted until death.

This case is interesting, as it shows the effect of a general infection and the rapidity with which the process adrances when it has once started.

CASE 3.-R. S. (permit No. 6701), schoolloy, aged 12 , entered Dec. 2, 1907, at 4:40 p. m. The patient gave a his. tory of falling off of a wagon three days previous to entrance. suffering a fracture of the tibia in the upper third, and a long, lacerated wound on the posterior surface of the calf of the left leg. The leg presented a sharply defined, bluish color extending from the ankle to the knee anteriorly and about 2 to 3 inches above the knee on the inner surface. The entire area was studded with blebs, from which a foul-smelling, greenish dis. charge was escaping. The wound had been closed on the ont. side by a physician. In the right groin was an area of discoloration 2 by 8 inches, and over the right great trochanter was an abrasion dressed with gauze covered over with collo. dion. Palpation of the left thigh and groin elicited distinct 
crepitation-emplrysema. The patient died December 3, at $4: 12$ in the morning, four days after the injury. Delirium in this case appeared on the third day and lasted until the end.

This case shorrs the effect of sealing dirty wounds and of not observing such a case closely. The clinical diagnosis was made, but there was no report of a bloodculture.

(AsE 4.-T. II., a packer, aged 51 (permit No. 5321), was admitted to the hospital Oet. 22. 1908, at $11: 05$ a. m. He entered the division suffering from an old. compound fracture of the radius and uhlua. nervous and very delirions. The diag. nosis at this tine was delirium tremens and compound frac. ture of the radius and ulna.

The arm was clark-brown in color, emplyysematous throughout, and a foul-smelling discharge was escaping. but no pus. The characteristic sweetish odor was present.

Later, $B . a$. c. infection was added to the diagnosis; bloodculture positive.

The patient died Oct. 22, 1908, at 12:50 p. m. Emergeney treatment was given, but could not be extensive in view of the fact that he was suffering from generalized infection.

The comment which I would make on this case is that it brings ont the point that the deliriuin and nervousness may be so great as to cause confusion as to whether or not the patient has delirium tremens.

('AsE 5.-D. C., a carriage-worker, aged 56 (permit No. 2657 ), admitted July 3, 1909 ; entered the St. Louis City Hospital with a history of severe traumatism, and as a result had a laceration of the head and right shoulder; the right hand was also badly crushed, showing evidence of pulpification, the dirt of the street being ground into the extremity. Bloodculture was positive. The patient died July 7, 1909, at $1: 20$ p. m., with delirium, nausea, vomiting, nervousness and all the signs of a general infection.

Consent to amputation was not obtained until the second day. Amputation was performed at the lower third of the right forearm with circular incision; skin approximated with thiree sutures. Drainage. A reduction of the shoulder was performed at this time. The next day ecchymosis was present about the wound. stitches were removed and thorough drainage instituted. On the fourth day, the forearm was badly swollen. discolored, crepitation of the tissues, bacteriologic examination showing presence of the gas bacillus. Incisions were made in the gangrenous area and the arm placed in a saline bath, the patient thoroughly septic.

This was a rase. evidently, in which the ecchymosis deceived the pliysician who was treating the case and in which a high amputation would probably have saved the life.

CASE 6...J. C. (permit No. 3395), a trackman, aged 45 , entered the hospital July 28, 1909, at 11 a. m., suffering from severe crushing injuries of both feet, produced by falling under a moving freight train. He.also had a fracture at the middle of the left leg.

Twenty-four homrs after entrance. the limb was amputated alout seven inches below the knee-joint ly the long posterior and short anterior flap method, with eareful ligation of vessels and resection of nerves. The skin-flaps were approximated with interrupted silkworm-gut sutures, with provisions for free drainage. The patient was in bad condition at the time of operation, lypodermoclysis being necessary. Moist dressing was applied.

Within thirty-six hours after admission. the symptoms of sepsis (namely, delirium, nausea, and vomiting) appeared. The patient climbed out of bed, and it was necessary to restrain lim. The following morning, signs of gas bacillus infection were very evident, the parts being dark, diwcolored, distended with gas, bubbles escaping, along with a black, bloody discharge. Blood-culture was positive. The antiseptics used in this ease were mercury bichlorid, $1 \cdot 2.000$. The patient died July :30. 1909), at $9: 40$ p. m.. nearly sixty hours after entrance.

Case 7.-F. T. (permit No. 5626), a laborer. aged 20, entered the hospital, Oct. 4,1509 . He gave a history of being hurt in a street-car accident. He had a crushing injury of the left thigh, was in extreme shock, and lis condition did not permit of immediate amputation.

'Two days after admission, his general condition had improved somewhat, although sepsis was extreme. The leg was very dark, beginning to become gangrenous. It was distended and full of gas. The patient was unable to stand a general anesthetic and, accordingly, with a long, sharp, amputation knife, the calf museles were severed at the point of fracture, about four inches below the knee-joint, vessels ligated and moist dressing applied. The patient died Oct. 8, 1909, four day's after admission.

Like those in the previous cases, this patient died with all the evidences of a general infection, his case being one of those in which the patient could not have stood a high amputation.

CASE. 8.-J. P. (permit No. 1868), aged 26, a driver, entered the hospital June 9, 1910, giving a history of being hurt in a runaway. The history of the accident as obtained later fron the patient was that the street was an improved one and was very muddy. Examination revealed three lacerated wounds on the middle third of the right leg, with a compound, com. minuted fracture of both bones of the left leg in the lower third, with numerous abrasions and contusions of the left foot. There were a few abrasions on the right leg.

Treatment at this time was directed to cleansing and shaving the limb, followed by an alcohol pack and iodin. The leg was placed on a cabot splint and 1.500 units of tetanus antitoxin administered.

Some twenty-four hours after patient's admission, the temperature rose, pulse became very rapid and the patient complained of some pain. Examination at this time revealed a dark, spreading inflammation, not sharply circumscribed, and without elevated borders. There was blel formation, and the leg presented signs of beginning gangrene. It was of a dusky color, or brownish lue, and in view of the fact that the patient's leg had been thoroughly rubbed in the dirty street, with the clinical signs as above, we believed that we were dealing with a gas bacillus infection. Cultures were taken, sent to the laboratory, and the following day were reported positive.

Without waiting for report from the laboratory, it was decided that. in order to give the patient a chance for his life. it would be necessary to do a high amputation at the junction of the lower and middle thirds of the thigh. Lnder a general ether anesthesia, the operation was performed by the circular cuff method. The wound was closed in layers overlapping the bone and two rubber tubes inserted for drainage. The patient left the table suffering from considerable shock. The wound was irrigated daily with hydrogen peroxid, and a hydrogen peroxid dressing applied. The process reached to the junction of the upper with the middle third of the leg, therefore it was ntcessary to go above the knee-joint.

The patient was discharged June 22, 1910, having made an uneventful recovery.

(ASE 9.-W. B. (permit No. 5877), aged 60. a pipecutter, entered the hospital Oct. 20, 1910, giving a history of having been injured by an elevator. Examination showed the right hand almost entirely torn from the arm at the carpal joint. The skin was torn loose around the entire wrist. All the muscles, fendons and nerves were severed, along with most of the vessels on the anterior surface, only those tendons on the posterior surface being intact. The hand was cold and sensation was abolished. The wound was cleansed and skin flaps loosely approxinated in the hope that there might be an establishment of circulation. Pulpification of the hand was great.

When first seen ly me two days after admission to the hospital, when the hand was dressed, it was found that the tissues presented an appearance very much like that of a gas bacillus infection. The laand was becoming dark in color: there was a foul-smelling, sweetish odor, with a dark, bloody discharge, and a few gas bubbles. Two hours after this. amputation was performed, the hand beginning to crepitate. The anteroposterior flap method was carried out at the junction of the lower with the middle third of the arm. On the following morning, after the operation, the patient's temperature was normal and he 
was doing well. Healing occurred by first intention, patient being discharged November 21 , after having remained in the hospital for three weeks preparatory to finding a home.

CASE 10.-G. Y. (permit No. 7853), a laborer, aged 25 , entered Dec. 31, 1910. The patient was suffering from a compound fracture of the forearm with laceration and erushing of forearm. There was considerable pulpification.

Temporary treatment was given. but in twenty-one hour. gas with a gangrenons process appeared. The forearm was very lark in color and gave off a foul. sweetish odor. There were gas bubbles in the tissues, as shown by crepitation and alwo by the excape of bubbles. The patient had a high temperature, rapid pulse, was septic and complained of consider able pain.

The patient was informed of his condition on three occasions. it heing necessary after thirty-six hours to bring the influence of his relatives to bear on him to obtain consent to operation. It was explained to him, at the time of operation, that the question of saving him was very doubtful on account of the delay.

Under general anesthesia, an anteroposterior flap at the junction of the upper and middle third was made in the usual way. Closure was with interrupted silkworm-gut sutures after irrigating the wound thoroughly with hydrogen peroxid. A rubber tube was inserted in each corner of the flap. and there after was daily irrigated with hydrogen peroxid and hydroser peroxid dressing was applied.

Within twenty-four hours, the patient's temperature had dropped to normal, followed in thirty-eight hours by a rise due to an infection of the wound with a probable staphylo. coecus infection. All sutures were removed, and hydrogen peroxid and tincture of iodin were freely used. Tlie wound. two weeks after amputation. was clean but required a repair amputation.

In this case, a gas-producing organism was found which in culture media produced gas, but an attempt to segregate it met with failure, although the dinisal eridence was unmistakably that of a gas bacillus infection with some contamination with the staphylococrens.

\section{CONClusions}

Farly interference, before serenty-two hours, preferably ammutation in malignant cases involving extremities, in most cases will mean the saving of life.

Taborers and railroad men are prone to infection, as their womds are often contaminated with soil.

Infertion occurs most frequently from contamination with street dirt, followed by railroad dirt, then elevator dist, and then intestinal tract discharges. The frequency of street soil infections, I believe can be explained on ground of contamination with manure. There are two recomed ases in which infection occurred in ischiorectal alocreses, the rarits, it is thonght. being due to the fact that these womds wenally are wide ouen and lined with a laver of gramulation tissue, the gramulation tissue prewenting the free dissection of the gas and the oxyen probably preventing the $B$. a. r. infection.

All wounds contaminated with street or railway dirt shomld he olserved for this infection and treated, if posible, in such a manner as to permit of easy inspecfim. Tonce plaster asts and sealing of wounds shomld bo aroirled. Cultures should be made if there is a suspicion of $B$. a. r. infection, and the wound freely opened and trealed with hydrogen peroxid or oxygen preparatory to amputation, if necessary. In Case 10, the amputation stmmp was irligated through drainage tubes with hydrogen peroxid twice daily for the firse two days, althongh at the time of operation the wound was flooded repeatedly with hydrogen peroxid.

Fall in temperature to normal would seem to indicate the pradication of $B$. a. c. infection, as in Cases 9 and 10
The disease is only relatively common, as it has occurred at the city liospital on an average of once in about 1,250 surgical cases, and practically always in cases in which there was pulpification of tissues. Since B. a. c. infections do not develop in very many or most cases of soil and street contaminated wounds (though often found here), it would seem that pulpification of tissue and injured blood-supply favor $B$. $a$ : $c$. infection, owing to weak or absent resistance. There is further erirlence to this effect, as shown in reported cases with presence of gas bacillus on and in amputation stumps.

Brewer has found that diabetics are prone to infection. and experimentally the $B, a . c$. derelop more easily and rapidly in the mesence of sugar.

Early diagnosis by definite, clean-cut, clinical pictures, and prompt, radical treatment will materially reduce the death-rate.

Blood-oultures should be made in every rase, but treatment should not wait.

I believe that the condition is often unrecognized, and trust that this report will stimulate an interest that will result in more lrequent diagnoses of the infection.

\section{A. IMPROVED APPARATUS FOR ESTIMAT- ING THE PRESSURE IN THE CERE- BROSPIXAL SYSTEM}

BLRRILL B. CROHN, M.D.

Volunteer Assistant. I'atbological Laboratory, Mount Sinai Hospital NEW YORK CITY

Since the introduction by Quincke ${ }^{1}$ of an apparatus for estimating the cerebrospinal pressure, there have been numerous criticisms of the mechanics of the instrument and several attempts have been marle, either to modify his apparatus, or to introduce a new principle. As devised by Quincke, the apparatus consists of the ordinary puncture needle and trocar. to which is attached a long piece of rubber tubing leading to an S-shaped piece of bent glass. According to the height to which the latter has to be raised the pressure is estimated, the reading being taken from the level of the hilt of the needle to the level of the fluid in the glass tube; this is measured by an independent centimeter scale.

The imperfections in the use of this instrument are the following:

First, betwen the time when the trocar is withdrawn from the needle and the moment when the rubber tubing is attached to the litit of the latter there must necessarily be an escape of fluid, even provicling that the worker is rapid in his actions and the connections are all firm and watertight. This loss of fluid, which is inevitable, and which may vary from 1 to 5 c.c. when the pressure is high, affects the accuracy of the rearling. secondly, the leneth of the rubber tubing and its usual lare caliber, (ances it to act in part as a reservoir, a long loop of this tubing frequently hanging far below the level of the needle. The latter, therefore, often withdraws from the estimation as much as 5 c.c. of fluid. When the pressure is low this interferes materially with the attempt to read intraspinal pressure.

The advantage of the instrument is that it permits a reading to be made before and after withdrawing the fluid, and allows examination of the fluid without dilution with another second inert fluid such as might be used in a manometer.

1. Quincke: Technik dir Lumbal Punction, 1902; Heutsch. Klin. 1906, i 351 